\title{
Comparison of Central Corneal Thickness Measurements Using Specular Microscope, Optical Biometer and Corneal Topographer
}

\author{
Madiha Waseem ${ }^{1}$, Mehvash Hussain ${ }^{2}$, Muhammad Muneer Quraishy ${ }^{3}$, Zaheer Sultan ${ }^{4}$ \\ ${ }^{1-4}$ Department of Ophthalmology, Dow University of Health Sciences, Karachi
}

\begin{abstract}
Purpose: To compare the Central Corneal Thickness (CCT) measurements by three different devices in normal eyes.

Study Design: Cross sectional observational study.

Place and Duration of Study: Dow University of Health Sciences and Dr. Ruth K.M. Pfau Civil Hospital, Karachi, from October 2020 to January 2021.

Methods: 80 eyes of healthy subjects aged between 20 to 50 years were included in the study. Patients with corneal pathologies, systemic disease, history of ocular surgery or trauma, high intraocular pressure and high refractive error were excluded. Subjects underwent full ophthalmic examination. Central Corneal Thickness was measured by specular microscope (Shin-Nippon SPM-700; Rexxam Co. Ltd, Takamatsu, Japan), optical biometer (AL-Scan; Nidek, Gamagori, Japan) and corneal topographer (TMS-5; Tomey corporation, Nagoya, Japan). All data entry and analysis was done on SPSS version 23. For correlation among devices, Pearson correlation coefficient was used. Scatter plot was drawn for graphical presentation.
\end{abstract}

Results: 80 eyes of 80 healthy subjects ( 50 males, 30 females) were recruited in the study by convenient sampling. The mean age was $37.76 \pm 8.35$ years. Mean Central Corneal Thickness values were $515.57 \pm 31.54$ $\mu \mathrm{m}, 510.21 \pm 30.11 \mu \mathrm{m}, 522.03 \pm 29.78 \mu \mathrm{m}$ with specular microscope, optical biometer and corneal topographer respectively. Measurements by these devices strongly correlate with each other using Pearson correlation coefficient $(r=0.927$ to $0.966, p \leq 0.001)$.

Conclusion: The results of Central Corneal Thickness measurements obtained from these three devices positively correlate with each other so any of these devices can be used for its measurement.

Key Words: Central Corneal Thickness (CCT), Specular microscope, Optical biometer, Corneal topographer.

How to Cite this Article: Waseem M, Hussain M, Quraishy MM, Sultan Z. Comparison of Central Corneal Thickness Measurements Using Specular Microscope, Optical Biometer and Corneal Topographer. Pak J Ophthalmol. 2021, 37 (3): 295-299.

Doi: $10.36351 /$ pjo.v37i3.1236

Correspondence to: Madiha Waseem

Department of Ophthalmology

Dow University of Health sciences

Karachi

Email: madiha.waseem@gmail.com

Received: March, 05, 2021

Accepted: April 28, 2021

\section{INTRODUCTION}

Corneal deturgescence by endothelial pump is indicated by corneal thickness. ${ }^{1}$ Normal central corneal thickness is $540 \mu \mathrm{m}^{2}$ Central Corneal Thickness evaluates corneal pathologies like keratoconus and corneal dystrophies. ${ }^{3}$ It is a key determinant of intraocular pressure and prevents misdiagnosis of glaucoma. ${ }^{4}$ Error of $3.4 \mathrm{~mm}$ of $\mathrm{Hg}$ in IOP measurement occurs with $10 \%$ difference in 
central corneal thickness. ${ }^{5}$ It evaluates cornea for refractive procedures. ${ }^{6}$ It is important in various disorders such as contact lens complications and diabetes mellitus. ${ }^{7}$ Various modalities are used for the measurement of corneal thickness. Contact methods include confocal microscopy and ultrasound Pachymetry. ${ }^{8}$ Noncontact methods such as topography, optical coherence tomography and specular microscopy are also used..$^{9}$ Corneal topography by Scheimpflug camera and scanning slit system provides corneal thickness map. ${ }^{10}$ Scheimpflug imaging devices include Tomey, Galilei, Pentacam, and Sirius. ${ }^{11}$ Optical biometer like AL-Scan uses diode laser of $830 \mathrm{~nm}$ and works on scheimpflug principle for central corneal thickness measurement. ${ }^{12}$ Specular microscope analyzes corneal endothelial cell count. It is also used for the measurement of corneal thickness. ${ }^{13}$ It uses light reflections to differentiate layers of cornea for the measurement of corneal thickness. ${ }^{14}$

The current study was undertaken to compare the central corneal thickness measurement by specular microscope, optical biometer and corneal topographer.

\section{METHODS}

This comparative study was done in the Department of Ophthalmology, Dow University of Health Sciences and Dr. Ruth K.M. Pfau Civil Hospital, Karachi from $15^{\text {th }}$ October 2020 to $30^{\text {th }}$ January 2021 . It included 80 right eyes of 80 healthy individuals aged 20 to 50 years, of both sexes, with refractive error of $\leq \pm 1.5$ diopters, healthy cornea and normal intraocular pressure of $\leq 21 \mathrm{mmHg}$ and normal fundus. This study adhered to the Declaration of Helsinki. Written and informed consent was obtained. All subjects underwent full ophthalmic examination including refraction, slit lamp biomicroscopy, measurement of intraocular pressure and fundoscopy. Exclusion criteria comprised of patients with corneal pathologies, systemic diseases such as diabetes mellitus, contact lens wearers, history of ocular surgery or trauma, intraocular pressure $>21 \mathrm{~mm} \mathrm{Hg}$ and refractive error $> \pm 1.5$ diopters.

Central Corneal Thickness (CCT) was assessed using specular microscope (Shin-Nippon SPM-700; Rexxam Co. Ltd, Takamatsu, Japan), optical biometer (AL-Scan; Nidek, Gamagori, Japan) and corneal topographer (TMS-5; Tomey corporation, Nagoya, Japan). All the readings were taken from the right eye by a single investigator in the morning between 10:00 am and 1:00 pm to avoid diurnal variation. Specular microscope determines corneal thickness in the range of $400-750 \mu \mathrm{m}$ by using light reflections from the anterior and posterior surface of the cornea. AL-Scan optical biometer uses scheimpflug imaging technique to measure central corneal thickness. Tomey corneal topographer TMS-5 combines scheimpflug and placido disc principle. It uses 25 - 31 rings capturing 256 point per ring and measurement time is around 1 second. The patients were asked to blink before each measurement and then fixate at the target after head positioning. Central zone of $3.0 \mathrm{~mm}$ of cornea was used. Three measurements were taken. The images were captured and analyzed with each device. An interval of 5 minutes was taken between measurements with the devices. Selection of devices was in random order as all of them were noncontact methods.

Data analysis was done on SPSS version 23. Qualitative data including gender was presented as frequency and percentage. Mean \pm standard deviation (SD) was calculated for age of the patients and Central Corneal Thickness (CCT). Pearson's correlation coefficient ( $r$ ) was used to show strength of relation among three devices for CCT measurement. R > 0.7 indicates strong positive correlation between devices. Scatter plot was used for graphical presentation of correlation among the three devices. $\mathrm{P} \leq 0.001$ was considered significant statistically.

\section{RESULTS}

The study included 80 eyes of healthy subjects. The age ranged from 20 to 50 years. The mean age was $37.76 \pm 8.35$ years. There were $50(62.5 \%)$ males and $30(37.5 \%)$ females. Table 1 shows Mean Central

Table 1: Central Corneal Thickness (CCT) measurements $(\mu \mathrm{m}), n=80$.

\begin{tabular}{lcc}
\hline Method & Mean & Standard Deviation \\
\hline Specular microscope & 515.57 & 31.545 \\
Optical biometer & 510.21 & 30.114 \\
Corneal topographer & 522.03 & 29.789 \\
\hline
\end{tabular}

Corneal Thickness (CCT) using different devices. There was strong positive correlation among all devices with Pearson correlation coefficient (r) more between specular microscope and optical biometer $(\mathrm{r}=$ $0.966)$ than between specular microscope and corneal topographer $(r=0.946)$ and between optical biometer 
and corneal topographer $(\mathrm{r}=0.927)$ as shown in Table 2. However, all three methods had strong correlation $(\mathrm{p} \leq$ 0.001). The scatter plots showed highest linear correlation $\left(\mathrm{R}^{2}\right)$ of CCT readings between specular microscope and optical biometer $\left(\mathrm{R}^{2}=0.934\right)$ in Figure 1 followed by the correlation between specular microscope and corneal topographer $\left(R^{2}=0.895\right)$ in Figure 2 and between optical biometer and corneal topographer $\left(\mathrm{R}^{2}=0.860\right)$ in Figure 3.

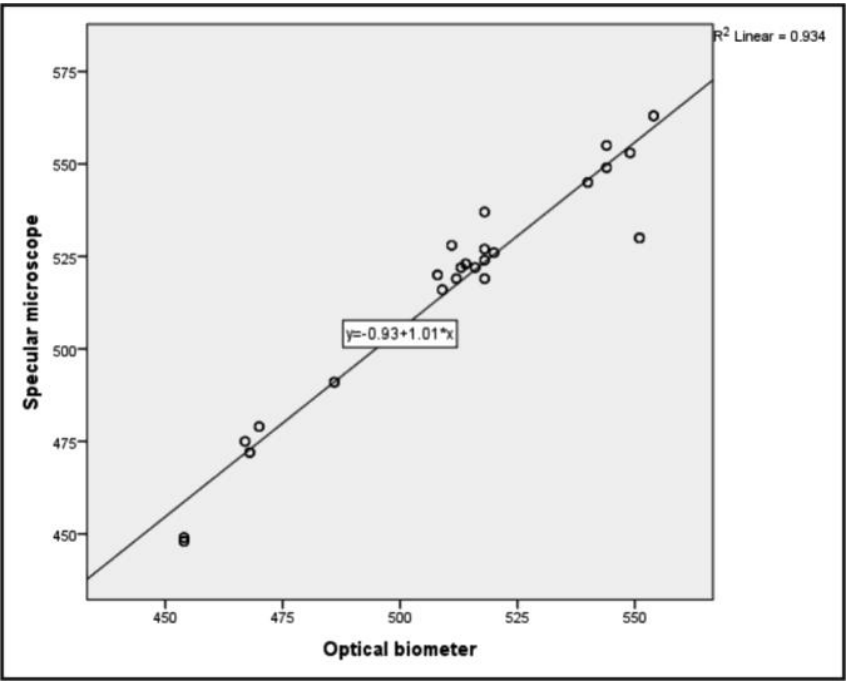

Figure 1: Scatter plot of central corneal thickness measurements by Specular microscope with Optical biometer.

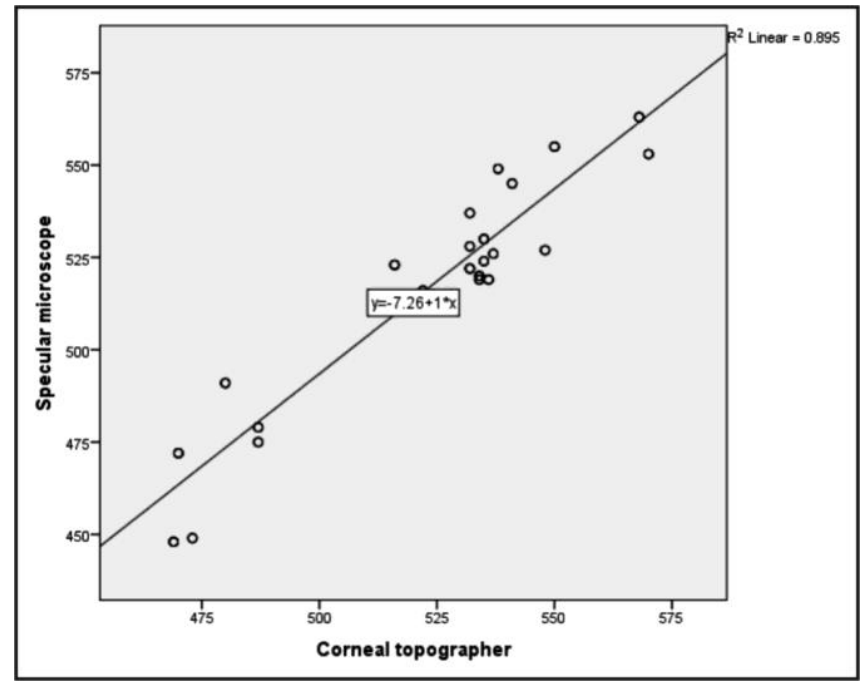

Figure 2: Scatter plot of central corneal thickness measurements by Specular microscope with Corneal topographer.
Table 2: Correlation among Specular microscope, Optical biometer and Corneal topographer.

\begin{tabular}{|c|c|c|c|c|}
\hline Method & & $\begin{array}{c}\text { Specular } \\
\text { Microscope }\end{array}$ & $\begin{array}{c}\text { Optical } \\
\text { Biometer }\end{array}$ & $\begin{array}{c}\text { Corneal } \\
\text { Topographer }\end{array}$ \\
\hline \multirow{3}{*}{$\begin{array}{l}\text { Specular } \\
\text { microscope }\end{array}$} & Pearson Correlation & 1 & $.966^{* * *}$ & $.946^{* * *}$ \\
\hline & Sig. (2-tailed) & & .000 & .000 \\
\hline & $\mathrm{N}$ & 80 & 80 & 80 \\
\hline \multirow{3}{*}{$\begin{array}{l}\text { Optical } \\
\text { biometer }\end{array}$} & Pearson Correlation & $.966^{* *}$ & 1 & $.927^{* *}$ \\
\hline & Sig. (2-tailed) & .000 & & .000 \\
\hline & $\mathrm{N}$ & 80 & 80 & 80 \\
\hline \multirow{3}{*}{$\begin{array}{l}\text { Corneal } \\
\text { topographer }\end{array}$} & Pearson Correlation & $.946^{* * *}$ & $.927^{* *}$ & 1 \\
\hline & Sig. (2-tailed) & .000 & .000 & \\
\hline & $\mathrm{N}$ & 80 & 80 & 80 \\
\hline
\end{tabular}

**. Correlation is significant at the 0.01 level (2-tailed).

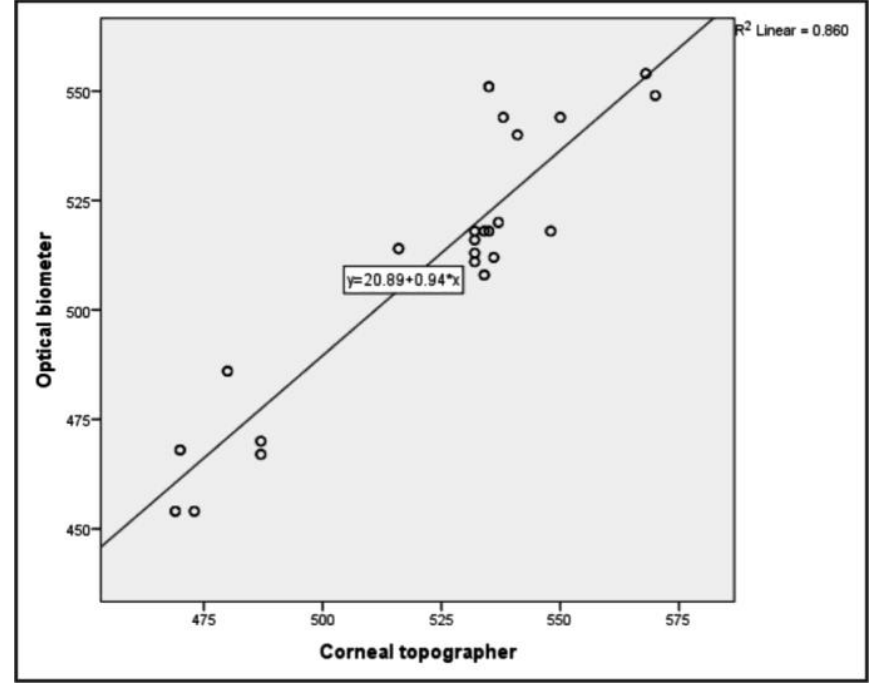

Figure 3: Scatter plot of central corneal thickness measurements by Optical biometer with Corneal topographer.

\section{DISCUSSION}

Measurement of CCT may be undertaken by ultrasonic pachymetry, confocal microscopy, corneal topography or optical coherence tomography. ${ }^{15}$ Central Corneal Thickness is an important component in the diagnosis of glaucoma and assessment of corneal disease. ${ }^{16}$ It is required for reliable preoperative assessment of candidates for keratorefractive surgery as corneal thickness of less than $500 \mu \mathrm{m}$ is a relative contraindication for LASIK. ${ }^{17}$ Increased corneal thickness may indicate early corneal decompensation. $^{18}$ Bourges et al observed that noncontact methods for CCT measurement can be used interchangeably with each other. ${ }^{19}$

In our study, mean CCT values were $515.57 \pm$ $31.54 \mu \mathrm{m}, 510.21 \pm 30.11 \mu \mathrm{m}, 522.03 \pm 29.78 \mu \mathrm{m}$ 
when measured by specular microscope, optical biometer and corneal topographer respectively. Different methods are available for Central Corneal Thickness (CCT) estimation and several studies have compared the accuracy of various devices. ${ }^{20}$ Mean CCT using scheimpflug analyzer was $536.4 \pm 35.77$ $\mu \mathrm{m}$ in a study conducted in Pakistani population which was comparable to our results and also reported positive correlation among different devices for CCT measurement $(\mathrm{r}=0.804$ to $\mathrm{r}=0.949) .{ }^{1}$ In a study by Chen et al, mean CCT with Scheimpflug imaging was $521.7 \pm 27.62 \mu \mathrm{m}$ which relates to our study. ${ }^{21}$ According to Sadik and Rahmi study, the mean CCT was $542 \pm 46 \mu \mathrm{m}$ with specular microscope. ${ }^{22}$ Other studies reported mean CCT of $518.53 \pm 34.96$ and $520 \pm 29$ with specular microscope which corresponds to our result. ${ }^{23,24}$ Jiang et al demonstrated that the mean CCT by specular microscope was 532.6. $\pm 40.0 \mu \mathrm{m}$. There was good correlation in scatter plot $(\mathrm{r}=0.954)$ between specular microscope and optical biometer. ${ }^{13}$ The mean CCT for the ALScan optical biometer and corneal topographer was $554.6 \pm 30.9 \mu \mathrm{m}$ and $570.7 \pm 30 \mu \mathrm{m}$ respectively. ${ }^{15}$

In the present study, there was strong linear correlation with Pearson correlation coefficient ranged from $r=0.927$ to $r=0.966$ when all three methods were compared. Many studies support our result. Ozyol et al in his study concluded that CCT measurements by optical biometer and Scheimpflug system are comparable with each other. ${ }^{25}$ Our study results are in accordance with the study by Reem which had positive correlation of CCT measurements between specular microscope and scheimpflug topographer $(\mathrm{r}=0.949) .{ }^{26} \mathrm{Khaja} \mathrm{W}$ et al, study found linear correlation between specular microscope $\left(\mathrm{r}^{2}=0.98\right)$ and corneal topographer $\left(\mathrm{r}^{2}=0.96\right)^{9}$. Luisa observed high correlation coefficient $(\mathrm{r}=0.852$ to 0.995) among different instruments for central corneal thickness. ${ }^{16}$

With the current pandemic of COVID-19 Corona virus disease, these methods of measuring Central Corneal Thickness are probably safer to use as all of them are non-contact methods.

Limitations of this study is the small sample size. Comparison between different age groups and different ethnic groups were also not made. Further study to address these issues are needed.

\section{CONCLUSION}

In normal eyes, CCT measurements by all three devices show strong linear correlation and these modalities can be used correspondingly for the measurement of central corneal thickness.

\section{Ethical Approval}

The study was approved by the Institutional review board/ Ethical review board.

(Ref: IRB-1787-DUHS/Approval/2020)

\section{Conflict of Interest}

Authors declared no conflict of interest.

\section{REFERENCES}

1. Qamar-ul-Islam SM. Comparison of central corneal thickness measurement using non-contact and contact pachymetry devices in normal eyes. Pak J Ophthalmol. 2015; 31 (1): 27-32.

2. Kanski J, Bowling B. Clinical Ophthalmology, 2016; Ed. $8^{\text {th }}$ : p. 168 .

3. Baghdasaryan E, Huang X, Marion K, Tepelus T, Bagherinia H, Sadda S, et al. Reproducibility of Central Corneal Thickness Measurements in Normal Eyes Using the Zeiss Cirrus 5000 HD-OCT and Pentacam HR. Open Ophthalmol J. 2018; 12 (1): 72-83.

4. Erdur S, Demirci G, Dikkaya F, Kocabora M, Ozsutcu M. Comparison of Central Corneal Thickness with Ultrasound Pachymetry, Noncontact Specular Microscopy and Spectral Domain Optical Coherence Tomography. Semin Ophthalmol. 2018; 33 (6): 782787.

5. Doughty MJ, Zaman ML. Human corneal thickness and its impact on intraocular pressure measures: a review and meta-analysis approach. Surv Ophthalmol. 2000; 44: 367-408.

6. Shafiq I, Hashmani S. Comparison Between Central Corneal Thickness Measurements Obtained with Orbscan II Topographer and Ultrasonic Pachymeter. Pak J Ophthalmol. 2007; 23: 87-90.

7. Faramarzi A, Ziai H. Central Corneal Thickness Measurement by Ultrasound versus Orbscan II. J Ophthalmic Vis Res. 2008; 3 (2): 83-86.

8. Akram S, Anklesaria ZH, Ahmad K. Correlation between Central Corneal Thickness Measurements Using Two Different Ultrasonic Pachymeters. Pak J Ophthalmol. 2013; 29: 214-216. 
9. Khaja W, Grover S, Kelmenson A, Ferguson L, Sambhav K, Chalam K. Comparison of central corneal thickness: ultrasound pachymetry versus slitlamp optical coherence tomography, specular microscopy, and Orbscan. Clin Ophthalmol. 2015; 9: 1065-1070.

10. Gonzalez-Perez J, Queiruga Pineiro J, Sanchez Garcia A, Gonzalez Meijome JM. Comparison of Central Corneal Thickness Measured by Standard Ultrasound Pachymetry, Corneal Topography, TonoPachymetry and Anterior Segment Optical Coherence Tomography. Curr Eye Res. 2018; 43 (7): 866-872.

11. Fan R, Chan TC, Prakash G, Jhanji V. Applications of corneal topography and tomography: a review. Clin Exp Ophthalmol. 2018; 46 (2): 133-146.

12. Huang J, Lu W, Savini G, Chen H, Wang C, Yu X, et al. Comparison between a New Optical Biometry Device and an Anterior Segment Optical Coherence Tomographer for Measuring Central Corneal Thickness and Anterior Chamber Depth. J Ophthalmology, 2016: 6347236. https://doi.org/10.1155/2016/6347236

13. Jiang J, Ong K. Variability of central corneal thickness measurements-comparing Zeiss IOL Master and Tomey corneal specular microscope. Asia Pac J Ophthalmol (Phila). 2019; 8 (4): 275-279.

14. Wu Q, Duan X, Jiang Y, Qing G, Jiang B, Shi J. Normal value of the central corneal thickness measured by non-contact specular microscope. Yan Ke Xue Bao. 2004; 20 (4): 229-232.

15. Can E, Eser-Ozturk H, Duran M, Cetinkaya T, Ariturk N. Comparison of central corneal thickness measurements using different imaging devices and ultrasound pachymetry. Indian J Ophthalmol. 2019; 67 (4): 496-499.

16. Pierro L, Iuliano L, Gagliardi M, Ambrosi A, Rama P, Bandello F. Central corneal thickness reproducibility among ten different instruments, Optom. Vis. Sci. 2016; 93 (11): 1371-1379.

17. Wang Z, Chen J, Yang B. Posterior corneal surface topographic changes after laser in situ keratomileusis are related to residual corneal bed thickness. Ophthalmology, 1999; 106 (2): 406-409.

18. Jeon HS, Hyon JY. Effects of Central Corneal Thickness on Early Postoperative Corneal Edema after Phacoemulsification. JSM Ophthalmol. 2016; 4 (2): 1044.

19. Bourges JL, Alfonsi N, Laliberté JF, Chagnon M, Renard G, Legeais JM, et al. Average 3-dimensional models for the comparison of Orbscan II and Pentacam pachymetry maps in normal corneas. Ophthalmology, 2009; 116 (11): 2064-71.
20. Sng C, Ang M, Barton K. Central corneal thickness in glaucoma. Curr Opin Ophthalmol. 2017; 28: 120-126.

21. Chen S, Huang J, Wen D, Chen W, Huang D, Wang Q. Measurement of central corneal thickness by highresolution Scheimpflug imaging, Fourier-domain optical coherence tomography and ultrasound pachymetry. Acta Ophthalmol. 2012; 90 (5): 449-455.

22. Cevik SD, Rahmi R, Cevik MT, Kivanc SA. Comparison of central thickness estimates by an ultrasonic pachymeter and non-contact specular microscopy. Arq Bras Oftalmol. 2016; 79 (5): 312-314.

23. Almubrad TM, Osuagwu UL, AlAbbadi I, Ogbuehi KC. Comparison of the precision of the Topcon SP3000P specular microscope and an ultrasound pachymeter. Clin Ophthalmol. 2011; 5: 871-876.

24. Ogbuehi KC, Osuagwu UL. Repeatability and interobserver reproducibility of Artemis - 2 high frequency ultrasound in determination of human corneal thickness. Clin Ophthalmol. 2012; 6: 761-769.

25. Ozyol E, Ozyol P. Comparison of central corneal thickness with four noncontact devices: an agreement analysis of swept-source technology. Indian J Ophthalmol. 2017; 65 (6): 461-465.

26. Azzam RI, Kasem MA, Khattab A, El-Fallal HM. Measurement of central corneal thickness by different techniques. J Egypt Ophthalmol Soc. 2017; 110: 114117.

\section{Authors' Designation and Contribution}

Madiha Waseem; Consultant Ophthalmologist: Concepts, Design, Literature search, Data acquisition, Data analysis, Statistical analysis, Manuscript preparation, Manuscript editing.

Mehvash Hussain; Assistant Professor: Data analysis, Statistical analysis, Manuscript preparation, Manuscript editing, Manuscript review.

Muhammad Muneer Quraishy; Professor: Design, Literature search, Manuscript preparation, Manuscript review.

Zaheer Sultan; Consultant Ophthalmologist: Search, Data acquisition, Data analysis, Manuscript review. 